\title{
Structural evolution of CatSper1 in rodents is influenced by sperm competition, with effects on sperm swimming velocity
}

\author{
Alberto Vicens, Maximiliano Tourmente and Eduardo RS Roldan*
}

\begin{abstract}
Background: Competition between spermatozoa from rival males for success in fertilization (i.e., sperm competition) is an important selective force driving the evolution of male reproductive traits and promoting positive selection in genes related to reproductive function. Positive selection has been identified in reproductive proteins showing rapid divergence at nucleotide level. Other mutations, such as insertions and deletions (indels), also occur in protein-coding sequences. These structural changes, which exist in reproductive genes and result in length variation in coded proteins, could also be subjected to positive selection and be under the influence of sperm competition. Catsper1 is one such reproductive gene coding for a germ-line specific voltage-gated calcium channel essential for sperm motility and fertilization. Positive selection appears to promote fixation of indels in the N-terminal region of CatSper1 in mammalian species. However, it is not known which selective forces underlie these changes and their implications for sperm function.

Results: We tested if length variation in the N-terminal region of CatSper1 is influenced by sperm competition intensity in a group of closely related rodent species of the subfamily Murinae. Our results revealed a negative correlation between sequence length of CatSper 1 and relative testes mass, a very good proxy of sperm competition levels. Since CatSper1 is important for sperm flagellar motility, we examined if length variation in the N-terminus of CatSper1 is linked to changes in sperm swimming velocity. We found a negative correlation between CatSper1 length and several sperm velocity parameters.
\end{abstract}

Conclusions: Altogether, our results suggest that sperm competition selects for a shortening of the intracellular region of CatSper1 which, in turn, enhances sperm swimming velocity, an essential and adaptive trait for fertilization success.

Keywords: CatSper1, Sperm competition, Indels, Positive selection, Sperm velocity, Rodents

\section{Background}

Many genes involved in sexual reproduction evolve rapidly, often as a result of adaptive evolution or positive selection [1]. Several selective forces have been proposed to drive the adaptive evolution of reproductive genes, including sperm competition, female cryptic choice, or sexual conflict [2-4]. Sperm competition arises when multiple males copulate with a female in a polyandrous system. As a consequence, the ejaculate of each male competes with those of other males to be the first to fertilize the egg(s) [4,5]. Sperm competition generates

\footnotetext{
* Correspondence: roldane@mncn.csic.es

Reproductive Ecology and Biology Group, Museo Nacional de Ciencias Naturales (CSIC), c/Jose Gutierrez Abascal 2, 28006 Madrid, Spain
}

postcopulatory sexual selection influencing reproductive traits to increase the success of an ejaculate at fertilizing under competitive conditions $[4,6]$.

A critical determinant of the outcome of sperm competition is the relative number of spermatozoa provided by different males. Males respond to sperm competition by increasing sperm numbers, which is achieved by an increase in testes mass relative to body mass [4]. Relative testes mass associates to levels of sperm competition in many taxa $[4,7-9]$ and, thus, is widely used as a reliable index of levels of sperm competition. Sperm swimming velocity is also a main determinant of fertilization success [10-13]. An increase in the size of sperm components is generally associated to increases in sperm swimming velocity [14-17]. 
Rapid divergence of reproductive proteins as a result of adaptive evolution may be linked to adaptive changes in reproductive traits under competitive conditions [1]. However, despite the potentially pervasive influence of sexual selection in driving adaptation at the molecular level, only a small set of studies has so far found an association between levels of sperm competition and rates of molecular evolution in reproductive genes [18-20]. In most studies searching for adaptively evolving proteins, the signature of positive selection has been tested estimating the ratio between nonsynonymous (amino acid-replacing) to synonymous (silent) nucleotide substitutions $(\mathrm{dN} / \mathrm{dS})$. Other forms of genetic variation different from nucleotide substitutions have received less attention in studies of adaptive evolution. One of these alternatives, i.e., structural variation, is widespread in animal genomes $[21,22]$ and structural variants may shape the variation in phenotypic traits between individuals [23]. Therefore, positive selection might also act setting structural variants in gene coding sequences which may be advantageous for certain physiological traits. Insertions and deletions (indels) have been shown to be subjected to positive selection in Drosophila accessory gland proteins [24]. Indel substitutions are the most abundant structural variants in the mammalian genome [21] and previous studies have reported that some mammalian reproductive proteins show an elevated fixation of indels [25-27], suggesting that positive selection may play a role in the evolutionary change of protein length.

CatSper is a sperm-specific $\mathrm{Ca}^{2+}$ ion channel that is exclusively found in the plasma membrane of the principal piece of the mammalian sperm flagellum, and presumably forms a heterotetrameric, $\mathrm{pH}$ - and voltage-dependent $\mathrm{Ca}^{2+}$-permeable channel [28]. CatSper channel allows $\mathrm{Ca}^{2+}$ influx into the sperm flagellum, which is important for sperm motility at different stages in the life of spermatozoa during their transit along the female tract to the site of fertilization. Differences may exist between species with regards to the timing and roles of CatSper activation, or in the mechanisms underlying motility regulation [29-32]. Thus, some evidence suggests that CatSper may be related to motion through viscous media such as that observed in the cervix or, perhaps, the utero-tubal junction, but not in later events during transit in the oviduct [31], and that CatSper channels are key elements of major $\mathrm{Ca}^{2+}$ entry pathways during basal (the so-called activated) motility [30]. On the other hand, targeted disruption of CatSper channel subunits results in a lack of a more vigorous (so-called hyperactivated) motility in mouse spermatozoa after incubation in conditions that prepare them for fertilization (i.e., capacitation) [29]. Mutant male mice have spermatozoa with reduced sperm velocity parameters after incubations and they are infertile [29]. This has led to the conclusion that CatSper is essential for sperm hyperactivation, which takes place in the oviduct, and is necessary for ova penetration during fertilization [28]. It is possible that CatSper activation can elicit functionally different behaviors according to extracellular $\mathrm{Ca}^{2+}$ concentrations and to the sensitivity of the sperm $\mathrm{Ca}^{2+}$ stores [31]. Altogether, because CatSper is important for sperm motility, and is required for male fertility, this channel is a promising candidate for a key involvement in sperm competition.

A high number of indel substitutions have been favored by positive selection in the first exon of the Catsper 1 gene, which codes for the intracellular $\mathrm{N}$-terminus of the CatSper channel $[25,26]$. Nevertheless, the selective forces underlying this high number of indels have not been identified. Although a clear function for this CatSper1 $\mathrm{N}$-terminus has not yet emerged, it is possible that the length of this region might affect the regulation of the CatSper channel. In such case, the structural variation of the N-terminus of CatSper1 could affect sperm flagellar motility and, as a consequence, influence sperm swimming velocity, which is a major determinant of reproductive success in sperm competition.

In this study, we examined whether an elevated rate of indels in the N-terminus of the CatSper1 sequence has an adaptive value in terms of sperm competition in rodents. First, we tested whether the indel-related length variation of the $\mathrm{N}$-terminal region of CatSper1 is associated to different levels of sperm competition. Second, given the vital role of the CatSper channel in sperm movement, and that sperm swimming speed is an important trait for fertilization, we assessed if changes in length of CatSper1 N-terminus are linked to phenotypic changes in sperm velocity parameters. Third, we analyzed whether sperm competition may promote episodes of positive selection at the nucleotide level. Fourth, since the amino terminus of CatSper1 may be involved in $\mathrm{pH}$ regulation of Catsper channel activity, due to its remarkably high content of histidine residues, we investigated whether the amount of histidines produced both by structural and molecular changes are associated with sperm competition and phenotypic adaptations. To this end, we sequenced the first exon of the Catsper 1 gene in several species belonging to the subfamily Murinae, assessed whether nucleotide and structural variations within this region may be driven by sperm competition, and examined possible associations between sequence length and sperm swimming velocity.

\section{Methods}

\section{Species}

Our study included a total of 16 rodent species belonging to the subfamily Murinae and comprising five genera: Mus spretus, Mus spicilegus, Mus macedonicus, Mus famulus, Mus caroli, Mus cookii, Mus pahari, Mus m. musculus, 
Mus m. domesticus, Mus m.castaneus, Mus m. bactrianus, Mus minutoides, Mastomys natalensis, Apodemus sylvaticus, Lemniscomys barbarus and Rattus norvegicus. This group of species covers a wide range of levels of sperm competition. Males of Mus species were purchased from the Institut des Sciences de l'Evolution, CNRS-Université Montpellier 2, France. Apodemus sylvaticus males were caught in the wild during the breeding session (Permit number 8688/02 from Consejería de Medio Ambiente, Comunidad de Madrid). Males of Mastomys natalensis and Lemniscomys barbarus come from wild-derived colonies which have been kept in captivity for only a few generations. Animal handling and housing followed the standards of the Spanish Animal Protection Regulation RD1201/2005, which conforms to European Union Regulation 2003/65. Animals were used complying with the Convention of Biological Diversity and the Convention on the Trade in Endangered Species of Wild Fauna and Flora. This study was approved by the Bioethics Committee of the Consejo Superior de Investigaciones Científicas (CSIC, Spain).

\section{Catsper 1 sequences}

The first exon of Catsper1 gene was amplified by polymerase chain reaction (PCR). PCR primers were designed based on the Catsper1 sequences published in the NCBI GenBank (http://www.ncbi.nlm.nih.gov) for Mus musculus and Rattus norvegicus (accession numbers NM_139301 and XM_001070492, respectively) as well as the genomic data for multiple mouse strains available from the Sanger Institute database (http:// www.sanger.ac.uk/). To design reverse primers, we also used Catsper1 coding sequences reported previously [26] for Mus species (GenBank accession numbers DQ021482DQ021500). These sequences were not employed to design forward primers because they started downstream of the start codon. PCR mixtures were prepared in a $50 \mu \mathrm{l}$ volume containing PCR Gold buffer 1× (Roche, Barcelona, Spain), $2.5 \mathrm{mM} \mathrm{MgCl}_{2}$ (Roche), $0.8 \mathrm{mM}$ dNTPs mix supplying $0.2 \mathrm{mM}$ of each deoxinucleotide triphosphate (Applied-Biosystems, Barcelona, Spain), $0.3 \mathrm{mM}$ of forward and reverse primers (Life Technologies, Madrid), $2 \mathrm{U}$ of DNA polymerase (Biotools, Madrid), and 20$200 \mathrm{ng} / \mu \mathrm{l}$ of genomic DNA template. All PCRs were performed in a Veriti thermocycler (Applied-Biosystems). The conditions of the thermocycler program consisted of $35-45$ cycles with an initial denaturation of $95^{\circ} \mathrm{C}$ for $30-40 \mathrm{~s}$, an annealing stage at $58-62^{\circ} \mathrm{C}$ (depending on folding temperature of primers) for $60 \mathrm{~s}$, and an elongation stage at $72^{\circ} \mathrm{C}$ for $80 \mathrm{~s}$. PCR products were purified by using the E.Z.N.A. ${ }^{\circ}$ Cycle Pure kit (Omega). Purified products were usually sequenced directly (Secugen S.L., Madrid, Spain). Products with problematic sequencing were cloned using pGEM-T Vector System (Promega, Madrid, Spain) following the protocol provided by the manufacturer. The first exon of Catsper 1 was sequenced for at least 3 individuals per species in order to generate a consensus sequence. Catsper1 sequences reported earlier [33] were not used to avoid polymorphisms due to different source populations.

\section{Alignments and trees}

Processing and correction of sequences were performed using the sequence viewer and alignment editor BioEdit. Sequences from several individuals belonging to the same species were used to generate a consensus sequence per species. Consensus sequences were bound to the coding sequence using as reference the sequence of Mus musculus retrieved from NCBI GenBank (accession number NM_139301). Nucleotide sequences were aligned using the algorithm ClustalW implemented in BioEdit. To test the robustness of the alignment, we performed repetitive ClustalW varying penalty parameters for gap opening and gap extension. Those regions in which indels lead to inaccurate alignments were manually edited. Nucleotide coding sequences were translated to amino acid sequences and the correct frame was checked using the protein sequence of Mus musculus, retrieved from NCBI GenBank (accession number NP_647462), as well as those translated sequences from Podlaha et al. [33]. Amino acid sequences were aligned through ClustalW.

CatSper1 phylogenies were reconstructed using NeighborJoining (MEGA 5.03) and Maximum Likelihood (PhyML) methods. Statistical selection of best-fit model of nucleotide substitution was performed by JModelTest software [33]. For evolutionary analyses we used an input tree comprising our range of species on the basis of well resolved phylogenies for rodents (see Results section) [34-37].

\section{Tests for positive selection}

We used the nonsynonymous/synonymous substitutions ratio $(\omega=\mathrm{dN} / \mathrm{dS})$ as an indicator of selective pressure at the protein level, with $\omega=1$ indicating neutral evolution, $\omega<1$ purifying selection, and $\omega>1$ diversifying positive selection. To estimate rates of sequence evolution we used the application Codeml implemented in the PAML 4 package. In order to detect variable selective pressures in the first exon of Catsper 1 and infer residues under positive selection we applied models that account for heterogeneous $\omega$ ratios among amino acid sites [38]. We compared a null model that does not allow sites with $\omega>1$ with a selection model that does through likelihood ratio tests. We used two kinds of likelihood ratio tests. The first compared a nearly neutral model M1a, which assumes values for $\omega$ between 0 and 1 , with a model M2a which allows values of $\omega>1$. The second test is more refined and compares two models assuming a beta distribution for $\omega$ values. In this 
case, the null model M7 that limits $\omega$ between 0 and 1 is compared to the alternative model M8, that adds an extra class of sites with an $\omega$ ratio estimated to be greater than 1. We also used a third test comparing the M8 model with the null hypothesis M8a, which fixes $\omega$ to 1 instead of estimating an additional class of sites, reducing false positives [39]. These tests compare twice the log-likelihoods of the alternative and the nullmodel to critical values from a chi-square distribution with the degrees of freedom equal to the difference in the number of parameters between the two models. If the alternative models showed a significantly better fit in the likelihood-ratio-test, Bayes empirical Bayes (BEB) analysis [40] was used to infer positively selected sites with posterior probabilities higher than 0.95 under both model M2a and M8.

To adjust codon frequency and number of gamma categories, we ran repetitive analysis varying the values of these parameters and we used the setting with the best fit according to the likelihood values of models.

\section{Estimation of lineage-specific evolutionary rates}

Site-analysis estimates variable $\omega$ ratios among sites and identifies residues under putative positive selection. Nonetheless, these models assume that the evolutionary parameters are invariant across the lineages of the phylogeny. We then calculated the evolutionary rates for each branch of the phylogeny using the Codeml free branch model [41]. Omegas were estimated for each lineage by adding $\mathrm{dN}$ and $\mathrm{dS}$ values from the root to the respective terminal branch and calculating the ratio of the sums. By calculating $\omega$ ratios from the root of the tree we considered the total accumulated selective pressures in Catsper1 during their evolution, which is more suitable for testing relationships against phenotypic data which do reflect the whole phenotypic evolution from the common ancestor [42]. In addition, estimating evolutionary data since the last common ancestor forces all branches to have the same length and therefore the analysis is not subject to temporal effects on $\mathrm{dN} / \mathrm{dS}$.

\section{Analysis of indel substitutions}

Indels produced in the first exon of Catsper1 were coded in the alignment by SeqState 1.41 software using a modified complex coding scheme. Events of indel substitutions were then inferred using the parsimony principle and considering the phylogenetic position of the species. This implies that, in cases where multiple equally parsimonious solutions for an indel were found, the first indel was assumed to happen in the split between the common ancestor of a clade with preponderance of an indel and the closest species carrying the indel variant. If the same indel variant was observed in any other species within the clade, such indel was considered homoplasious by evolutionary convergence. The species Cricetulus griseus was used as outgroup to infer indels occurring between Rattus norvegicus and remaining lineages. Indels were identified either as deletions or insertions and they were mapped onto the species tree (see Results section).

Length of the N-terminus of CatSper1 was calculated for each species as the total number of amino acids after checking that no indel is produced in the flanking regions and thus assuming that variations in sequence length are exclusively driven by internal indels.

\section{Analysis of histidines}

Total number and proportion of histidines were calculated for each species to assess whether molecular and structural changes are promoting variations in the amount of this residue which is presumably important for the regulation of the CatSper channel.

\section{Sperm competition and sperm velocity parameters}

Relative testes mass has been widely used as a reliable indicator of sperm competition levels in analyses of the evolution of ejaculate traits [9] and reproductive genes $[18,20,43]$. To obtain relative testes mass, males $(\mathrm{N}=5$ for each species) were sacrificed by cervical dislocation and weighed. After removal, the testes were weighed and measured. Values of relative testes mass for Rattus norvegicus were taken from the literature [44]. Mean relative testes mass values were calculated using the regression equation for rodents [44] (Additional file 1).

Sperm velocity parameters were measured using a computer-assisted sperm analyzer (Sperm Class Analyzer v.4.0, Microptic, Barcelona, Spain). A total of $5 \mu \mathrm{l}$ of sperm suspensions was placed in a $20-\mu \mathrm{m}$ deep slide chamber (Standard Count-2 Chamber Slide 20-micron, Leja, Nieuw-Vennep, Netherlands) pre-warmed to $37^{\circ} \mathrm{C}$, and examined using phase contrast microscopy with a $4 \times$ objective. Data on sperm velocity parameters were obtained within $5 \mathrm{~min}$ of sample collection for all individuals.

Using a video camera (Basler A312fc, Vision Technologies), up to eight videos of $4 \mathrm{~s}$ each were recorded for each male's sperm sample. Sperm concentration was previously adjusted to $4-6 \times 10^{6} \mathrm{sperm} / \mathrm{ml}$ to satisfy the requirements of the analysis. Videos were analyzed and a minimum of 150 tracks were obtained for each male's sample, with $\mathrm{N}=5$ males analyzed for each species.

Seven sperm velocity parameters were quantified: curvilinear velocity (VCL) (in $\mu \mathrm{m} / \mathrm{s}$ ), straight line velocity (VSL) (in $\mu \mathrm{m} / \mathrm{s}$ ), average path velocity (VAP) (in $\mu \mathrm{m} / \mathrm{s}$ ), linearity (LIN) (in\%), straightness (STR) (in\%), amplitude of lateral head displacement (ALH) (in $\mu \mathrm{m} / \mathrm{s}$ ) 
and beat cross frequency (BCF) (in $\mathrm{Hz}$ ). To reduce potentially correlated components of velocity to a single factor summarizing the information, we performed a principal component analysis (PCA). The seven velocity descriptors were used as variables in the PCA, rendering two principal components that accounted for 70\% (PC1) and $22 \%$ (PC2) of total variability. The three principal sperm velocity parameters (VCL, VSL, and VAP) showed a significant positive correlation with $\mathrm{PC} 1$ and no correlation with $\mathrm{PC} 2$, and thus $\mathrm{PC} 1$ was interpreted as the global measure of sperm velocity (hereafter referred as "overall sperm velocity").

\section{Statistical analyses}

Possible relationships between the evolution of the $\mathrm{N}$-terminal region of CatSper 1 and phenotypic and ecological adaptations were evaluated through linear regression analyses. To test whether evolutionary dynamics of N-terminus of CatSper 1 are associated with sperm competition, linear regression analyses were performed using $\omega$ calculated from the root of the phylogeny and sequence length as dependent variables and relative testis mass as predictor variable. The same dependent variables were used in multiple regression analyses with body mass and testes mass as predictor variables. In this case, since predictor variables are related to each other, they were added to the multiple regression analysis in order, first body mass, and then testes mass, using a sequential (Type I) sum of squares. In order to search for relationships between CatSper 1 evolution and sperm velocity, different descriptors of sperm swimming velocity (see above) were used as dependent variables in simple regression analysis with $\omega$ and CatSper1 sequence length as predictor variables. The level of significance was adjusted to $P<0.05$ for all tests. Mus m.bactrianus, Mus famulus and Mus cookii were not included in these analyses because of lack of data for these species.

Since species may share character values as a result of a common ancestry rather than independent evolution, regression analyses were performed using a phylogenetic generalized least-squares (PGLS) approach [45]. This powerful method allows for a control of phylogenetic effects on the associations between variables. PGLS analyses were conducted using the CAPER package for the statistical environment R v.2.10.1 (R Development Core Team, 2011). Phylogenetic effects were controlled based on the tree topology and branch lengths were calculated under the M0 model included in PAML.

\section{Results}

\section{Catsper1 sequences}

Nucleotide sequences of the first exon of the Catsper1 gene were obtained for 16 murid species and aligned according to their coding sequences. Multiple ClustalW alignment spanned 1080 nucleotides and revealed a high sequence divergence as well as an elevated number of gaps in Catsper1 (see Additional file 2). Sequences among species varied in length from 879 to 957 nucleotides. Amino acid alignment of the N-terminal region of CatSper1 is shown in Figure 1.

Catsper1 phylogenies built by Neighbor-Joining and Maximum Likelihood approaches (Additional file 3) showed almost identical topology, but they showed some differences when compared to the species tree (Figure 2), which suggests that CatSper1 may be subjected to selective forces that would alter the evolutionary pattern expected from the phylogenetic relationships among the species. A total of 58 parsimony-inferred indel substitutions in the first exon of Catsper1 were mapped onto the species tree (Figure 2). A total of 50 of these indels were unique and congruent with the tree topology and 8 were homoplasious (Figure 2). No indel polymorphism was found between individuals, by which we assume that all consensus sequences are representative of each species. A total of 35 indels fell in terminal branches and ranged from none for Mus cokii, Mus spretus, Mus spicilegus, Mus macedonicus, Mus m. domesticus and Mus m. castaneus to 9 for Mastomys natalensis (Figure 2). A total of 34 deletions were detected throughout the phylogeny against 24 insertions.

All indels identified in the alignment spanned lengths of $3 \mathrm{n}$ nucleotides (see Figure 2 and Additional file 2), and thus the reading frame remained intact in all sequences. The number of observed indels in the first exon of Catsper1 was significantly higher than both the genomic average and Catsper 1 locus neutral indel substitution rates (see [33] for methodology).

\section{Tests for positive selection}

A high number of amino acid replacements was observed in CatSper1 $\mathrm{N}$-terminus in addition to indels (Figure 1), suggesting that positive selection could also be promoting molecular variation in Catsper1. Robust evidence of positive selection was detected in the first exon of Catsper 1 when likelihood values of M2a and M8 selection models were compared with the corresponding values of M1a, M7 and M8a neutral models (Table 1). In both cases, likelihood ratio tests rejected the neutral models. A total of 10 and 9 significant positively selected sites were identified with a Bayesian posterior probability of $P_{\mathrm{B}}>0.95$ under M2a and M8 models respectively (Table 1, Figure 1). These analyses reveal that the Nterminal region of CatSper1 is subjected to strong positive selection also at the molecular level.

We found that 4 out of 10 (40\%) positively selected sites fell in positions containing histidines. Considering the possible role of histidine residues as a $\mathrm{pH}$-sensor in 


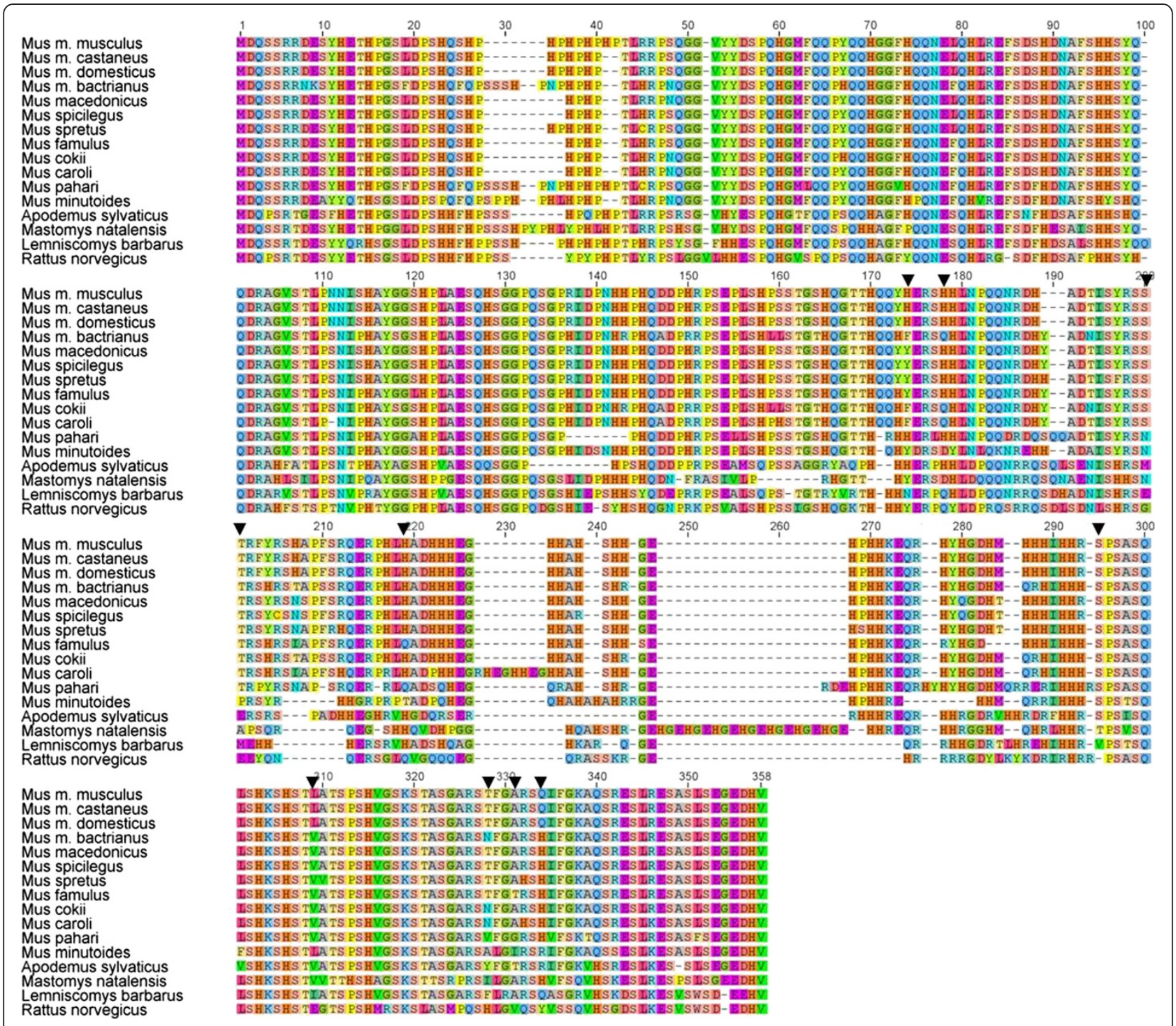

Figure 1 Amino acid alignment of $\mathbf{N}$-terminal region of CatSper1. Translated sequences of 16 rodent species analyzed in this study. Dashes represent alignment gaps. Arrows on positions represent sites under putative positive selection with a Bayesian posterior probability $>0.95$ under M2a and M8 models.

CatSper1 activation, adaptive mutations on this domain may have important functional implications.

\section{CatSper1 evolution and sperm competition}

Lineage-specific evolutionary rates $(\omega)$ estimated under PAML free branch model for the first exon of Catsper 1 were greater than 1 in all cases except for Lemniscomys barbarus (Additional file 1), thus revealing that intense positive selection is acting on CatSper among these rodent species. To seek evidence for an influence of sperm competition on the evolutionary rate of CatSper1, we correlated lineage-specific $\omega$ ratios with their respective relative testes mass values. Phylogenetic generalized leastsquares (PGLS) analysis correcting for phylogenetic effects showed no significant associations of lineage-specific $\omega$ with relative testes mass or testes mass corrected for body mass (Table 2).

To test whether structural variation of CatSper1 Nterminal region is influenced by sperm competition, correlations between length of this region and relative testes mass were examined. Because the PGLS test is a least squares-based regression analysis, and it is highly sensitive to violations of assumptions and outliers, we first searched for the presence of outliers in our dataset. We found that sequence length of Mastomys natalensis was very different from that of the other species (Additional file 4). This was mainly due to its specific 51-bp long insertion produced as the result of a recent tandem 


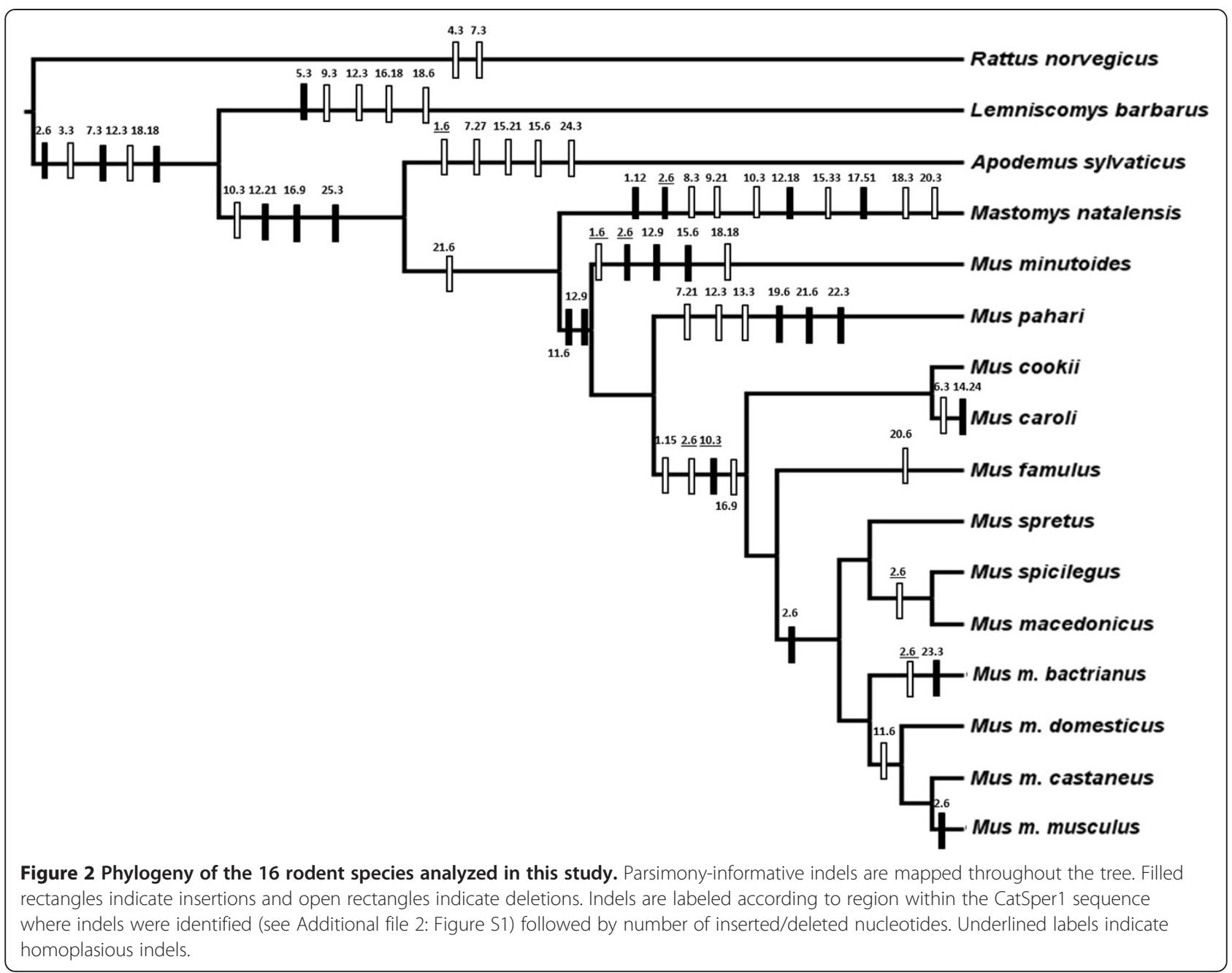

duplication containing 3 codons (see Figure 1, Additional file 2). Therefore, we have not included this lineage in the analyses. A significant negative correlation was observed between the length of CatSper1 N-terminus and relative testes mass (Table 2). Species with high values of relative testes mass (i.e., with higher levels of sperm competition) presented a shorter N-terminus of CatSper1 whilst those with lower values of relative testes mass (low intensity of sperm competition) showed longer fragments (Figure 3).

Table 1 Tests of positive selection in CatSper 1

\begin{tabular}{|c|c|c|c|c|c|}
\hline $\mathbf{N}$ & Ls & $\begin{array}{l}\text { Best fit } \\
\text { model }\end{array}$ & $\begin{array}{l}\text { Log-likelihood } \\
\text { values }^{a}\end{array}$ & Parameter estimates & PSS $^{\mathbf{b}}$ \\
\hline \multirow[t]{5}{*}{16} & 235 & M1a & -2907.9808 & $\mathrm{p}_{0}=0.269, \mathrm{p}_{1}=0.731, \omega_{0}=0.027, \omega_{1}=1$ & Not allowed \\
\hline & & $\mathrm{M} 2 \mathrm{a}$ & $-2879.567332 * *$ & $\begin{array}{l}p_{0}=0.186, p_{1}=0.651 p_{2}=0.163, \omega_{0}=0 \\
\omega_{1}=1, \omega_{2}=4.67\end{array}$ & $\begin{array}{l}124 \mathrm{H}^{*}, 128 \mathrm{H}^{*}, 146 \mathrm{~S}^{*}, 147 \mathrm{~T}^{* *}, 154 \mathrm{H}^{* *}, 165 \mathrm{~S}^{*}, \\
179 \mathrm{~L}^{*}, 198 \mathrm{~T}^{* *}, 201 \mathrm{~A}^{* *}, 204 \mathrm{Q}^{* *}\end{array}$ \\
\hline & & M7 & -2908.013308 & $p=0.041, q=0.012$ & Not allowed \\
\hline & & M8 & $-2879.676624 * *$ & $\begin{array}{l}p_{0}=0.833, p=0.0166, q=0.0052, p_{1}=0.166 \\
\omega=4.535\end{array}$ & $\begin{array}{l}124 \mathrm{H}^{*}, 146 \mathrm{~S}^{*}, 147 \mathrm{~T}^{* *}, 154 \mathrm{H}^{* *}, 165 \mathrm{~S}^{*}, 179 \mathrm{~L}^{*}, \\
198 \mathrm{~T}^{* *}, 201 \mathrm{~A}^{* *}, 204 \mathrm{Q}^{* *}\end{array}$ \\
\hline & & M8a & -2907.982933 & $\begin{array}{l}p_{0}=0.269, p=2.758, q=99.0, p_{1}=0.731 \\
\omega=1.0\end{array}$ & Not allowed \\
\hline
\end{tabular}

${ }^{a}$ Differences between log-likelihood values of models with $99 \%$ statistical significance level for 2 degrees of freedom are shown as **.

${ }^{\text {b }}$ Positively selected sites (PSS) with a posterior probability $>0.95\left(^{*}\right)$ and $>0.99\left(^{* *}\right)$ in Bayes Empirical Bayes.

$\mathrm{N}$, Number of sequences aligned.

Ls, Sequence length (in codons) after alignment gaps are removed.

Parameter estimation and likelihood scores under site models. 
Table 2 PGLS analyses of evolutionary rate and sequence length of CatSper 1 in relation to relative testis mass (RTM)

\begin{tabular}{|c|c|c|c|c|c|c|c|c|c|c|c|c|c|}
\hline Dependent variable & Predictor & $\mathrm{n}$ & d.f. & Slope & $R^{2}$ & $F$ & $\mathbf{P}$ & $\lambda^{a}$ & $P_{(\lambda=0)}^{b}$ & $P_{(\lambda=1)}^{b}$ & $\mathrm{ES}^{\mathrm{c}}$ & $\mathrm{CL}(-)^{\mathrm{d}}$ & $\mathrm{CL}(+)^{\mathrm{d}}$ \\
\hline Omega & RTM & 16 & 14 & -0.049 & 0.012 & 0.139 & 0.855 & 0.999 & $<0.001$ & 1 & 0.106 & -0.438 & 0.649 \\
\hline \multirow[t]{2}{*}{ Omega } & Body mass & 16 & 14 & 0.0022 & 0.539 & 13.939 & 0.0028 & 0.999 & $<0.001$ & 1 & 0.052 & -0.363 & 1.449 \\
\hline & Testes mass & & & -0.0924 & & 0.14 & 0.7144 & & & & 0.056 & -0.439 & 0.647 \\
\hline Sequence length & RTM & 15 & 13 & -7.214 & 0.53 & 14.682 & $0.002^{* *}$ & 0.402 & 0.257 & 0.078 & 0.925 & 0.359 & 1.491 \\
\hline \multirow[t]{2}{*}{ Sequence length } & Body mass & 15 & 12 & 0.149 & 0.778 & 8.895 & $0.011^{*}$ & 0 & 1 & 0.007 & 0.779 & 0.214 & 1.345 \\
\hline & Testes mass & & & -21.159 & & 33.055 & $0.0009^{* *}$ & & & & 1.28 & 0.714 & 1.846 \\
\hline
\end{tabular}

$P$ values $<0.05\left(^{*}\right)$ and $<0.01\left(^{* *}\right)$ indicate statistical significance.

${ }^{a} \lambda$ value indicates phylogenetic effect.

${ }^{\mathrm{b}}$ Significance of log-likelihood ratios for $\lambda$ against models with $\lambda=0$ and $\lambda=1$ are shown. Statistically significant values are shown in bold.

${ }^{\mathrm{c}}$ Effect size (ES) calculated from the $F$-values.

${ }^{d}$ Noncentral $95 \%$ confidence limits (CL). Confidence intervals excluding 0 indicate statistically significant relationships and are shown in bold.

\section{CatSper1 evolution and sperm swimming velocity}

Given the important role of CatSper in sperm motility, we evaluated whether molecular and structural divergence of its $\mathrm{N}$-intracellular domain has effects on sperm movement properties. No significant relationships were obtained when evolutionary rates of Catsper 1 were correlated with parameters of sperm velocity (data not shown). On the other hand, significant negative correlations were obtained when CatSper1 N-terminus length was analyzed with parameters measuring linear velocity (VSL, VAP, LIN, STR) and an overall sperm velocity component estimated from PCA (Table 3, Figure 4).

\section{Changes in histidine residues}

Because of its high histidine content, the $\mathrm{N}$-intracellular region of CatSper1 is thought to be involved in the $\mathrm{pH}$ mediated regulation of the CatSper channel. Therefore, we evaluated whether the variation in histidine content

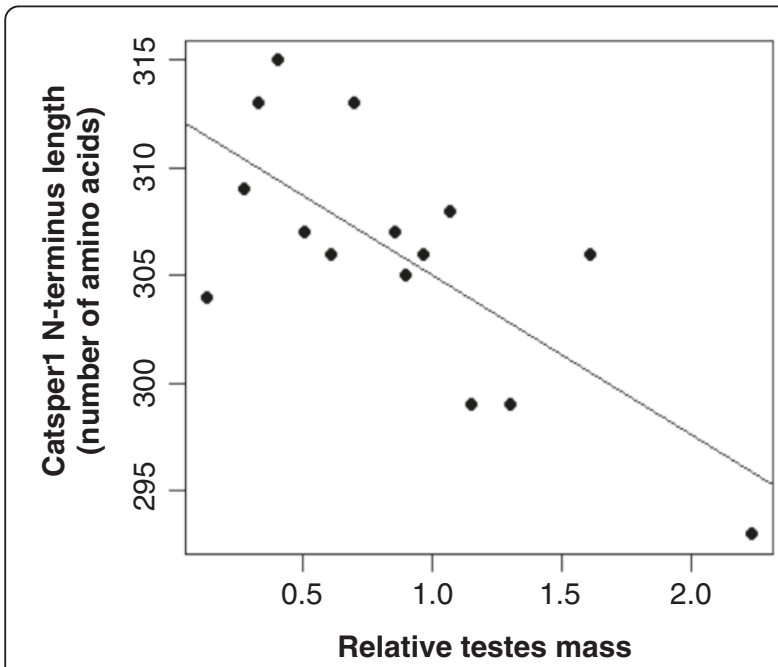

Figure 3 Relationship between relative testes mass and the length of CatSper1 $\mathrm{N}$-terminal region in rodents. Results of statistical analyses are given in Table 2. observed across rodent species is related to levels of sperm competition. We calculated the proportion of histidines in each sequence (Additional file 1) and performed PGLS analysis with relative testes mass as predictor; we found no significant correlation $(F=1.47$, $P=0.25$ ) (Additional file 5A). Likewise, no significant correlation was found between the proportion of histidines and parameters of sperm swimming velocity (data not shown).

These results suggest that sperm competition has no influence in histidine content of rodent CatSper1. No significant relationship was observed between sequence length and histidine proportion $(F=3.26, P=0.0941)$. Thus, differences in histidine content are not attributable to residues gains and losses by insertions and deletions. Instead, two global histidine gains were detected throughout the phylogeny: the first occurring in the common ancestor of murid species after the split from rat, and a second between Mus pahari and the remaining Mus species (Additional file 5). These observations suggest that the proportion of histidines in CatSper1 is evolving following a phylogenetic pattern.

\section{Discussion}

Previous studies in primates and rodents revealed that the rate of indel substitutions in the first exon of Catsper 1 is higher than that in neutral genomic regions, suggesting that positive selection is promoting length variation in CatSper1 $[25,26]$. Because CatSper1 is a possible candidate for direct involvement in sperm competition (see Background), we assessed whether the fixation of indel substitutions in the $\mathrm{N}$-terminal region of CatSper1 is associated to this selective force. Our results revealed a significant negative correlation between the length of CatSper1 N-terminus and relative testes mass, a reliable proxy of sperm competition. This finding provides evidence that sperm competition has an influence on structural dynamics of CatSper and favors a shortening of the $\mathrm{N}$-terminal domain of this protein. It has been 
Table 3 PGLS of CatSper1 length in relation to sperm velocity parameters

\begin{tabular}{|c|c|c|c|c|c|c|c|c|c|c|c|c|c|}
\hline Dependent variable & Predictor & $\mathbf{n}$ & d.f. & Slope & $\mathbf{R}^{2}$ & $\mathrm{~F}$ & $\mathbf{P}$ & $\lambda^{a}$ & $P_{(\lambda=0)}^{b}$ & $\mathrm{P}_{(\lambda=1)}^{\mathrm{b}}$ & $\mathrm{ES}^{\mathrm{c}}$ & $\mathrm{CL}(-)^{\mathrm{d}}$ & $\mathrm{CL}(+)^{\mathrm{d}}$ \\
\hline VCL & Sequence length & 11 & 9 & -0.975 & 0.512 & 1.966 & 0.191 & 0.754 & 0.066 & 0.362 & 0.430 & -0.223 & 1.083 \\
\hline VSL & Sequence length & 11 & 9 & -2.375 & 0.216 & 10.480 & $0.0089^{* *}$ & 0.667 & 0.186 & 0.103 & 0.898 & 0.245 & 1.551 \\
\hline VAP & Sequence length & 11 & 9 & -2.003 & 0.164 & 8.340 & $0.016^{*}$ & 0.556 & 0.329 & 0.110 & 0.818 & 0.165 & 1.472 \\
\hline ALH & Sequence length & 11 & 9 & 0.044 & 0.201 & 2.265 & 0.159 & 0.528 & 0.943 & 0.188 & 0.483 & -0.21 & 1.176 \\
\hline LIN & Sequence length & 11 & 9 & -0.016 & 0.785 & 32.795 & $0.003^{* * *}$ & 0 & 1 & 0.039 & 1.402 & 0.709 & 2.095 \\
\hline STR & Sequence length & 11 & 9 & -0.009 & 0.626 & 15.06 & $0.001^{* * *}$ & 0.66 & 0.175 & 0.161 & 1.074 & 0.381 & 1.767 \\
\hline$B C F$ & Sequence length & 11 & 9 & 0.016 & 0.009 & 0.085 & 0.919 & 1 & 0.001 & 1 & 0.097 & -0.595 & 0.789 \\
\hline Overall Sperm Velocity & Sequence length & 11 & 9 & -0.195 & 0.475 & 10.03 & $0.005^{* *}$ & 0.652 & 0.128 & 0.154 & 0.920 & 0.227 & 1.613 \\
\hline
\end{tabular}

$P$ values $<0.05\left(^{(*)},<0.01\left(^{* *}\right)\right.$ and $<0.005\left(^{* * *}\right)$ indicate statistical significance.

${ }^{\mathrm{a}} \lambda$ value indicates phylogenetic effect.

${ }^{\mathrm{b}}$ Significance of log-likelihood ratios for $\lambda$ against models with $\lambda=0$ and $\lambda=1$ are shown. Statistically significant values are shown in bold.

'Effect size (ES) calculated from the $F$-values.

${ }^{\mathrm{d}}$ Noncentral $95 \%$ confidence limits (CL). Confidence intervals excluding 0 indicate statistically significant relationships and are shown in bold. VCL, Curvilinear velocity; VSL, Straight-line velocity; VAP, Average path velocity; ALH, Amplitude of lateral head displacement; LIN, Linearity; STR, Straightness; BCF, Beat cross frequency.

reported that variation in molecular mass of SvsII, the major component of rodent copulatory plug, correlates positively with sperm competition levels across rodent species [27]. Given that most of the variation in the molecular mass of SvsII is presumably produced through insertions and deletions, this was so far the only evidence suggesting that indels may explain adaptive length divergence in reproductive proteins under scenarios of sperm competition. Our results now provide strong support for the idea that sexual selection is responsible for structural variation in some reproductive proteins.

The mechanisms generating insertions and deletions in the genome are not well understood. In any case, it

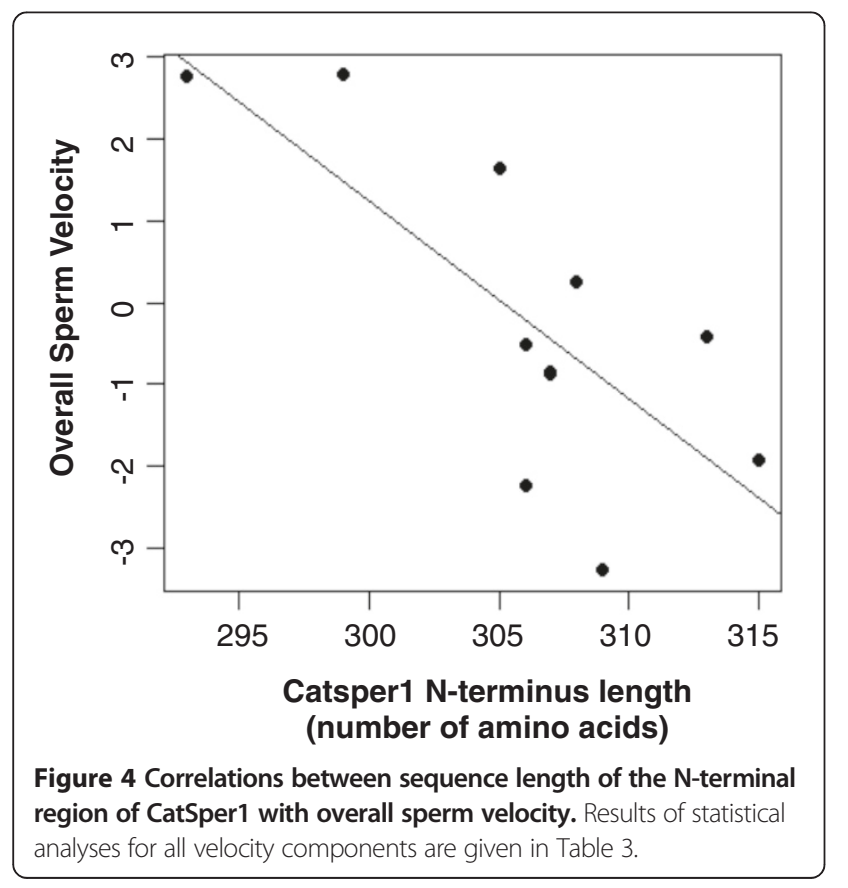

has long been recognized that small deletions are more abundant than insertions of similar size in proteincoding sequences [46]. Our results agree with this evidence, because deletions outnumbered insertions in CatSper1. Moreover, we observed a variable number of indels across terminal branches, with some lineages showing insertion:deletion ratios that deviated from the expected ones. The most extreme cases were the species subjected to more intense sperm competition, namely Apodemus sylvaticus and Lemniscomys barbarus which showed almost an exclusive presence of deletions. It seems, therefore, that a higher fixation of deletions in the first exon of Catsper 1 is not only resulting from deletion bias as indel mechanism, but also that selective forces such as sperm competition are contributing to the shortening of this region.

CatSper1 is important for the regulation of intracellular $\mathrm{Ca}^{2+}$ required for both activated and hyperactivated sperm motility [29-31] that is needed at different stages during transit in the female tract. Experiments using null mice revealed that CatSper1 is essential for maintenance of sperm motility and hyperactivation [29,30], maintenance of intracellular ATP levels [47], progression beyond the oviductal sperm reservoir [48] and, ultimately, male fertility $[29,49]$. We thus tested whether length variation in the $\mathrm{N}$-terminal region of CatSper1 is linked to sperm swimming velocity. Our results showed a significant negative correlation of CatSper1 $\mathrm{N}$-terminus length with four parameters describing velocity (VSL, VAP, STR and LIN) and an overall sperm velocity component, revealing an association between the shortening of this region and increases in progressive motility. Our findings thus support a relationship between structural divergence of CatSper1 and sperm movement among rodent species. Previous studies have shown evidence that when sperm competition increases so does sperm swimming speed 
$[14,17,50]$. Swimming speed is a major determinant of fertilization success in sperm competition contexts $[10,11]$. Based on the correlation observed between relative testes mass, CatSper1 sequence length, and sperm velocity parameters, our results support the hypothesis that sperm competition favors shortening in the $\mathrm{N}$-intracellular domain of CatSper1 leading to an increases of sperm swimming speed during transport of spermatozoa in the female reproductive tract and, as a consequence, increase the probabilities of fertilization success.

Although a high number of amino acid replacements, driven by positive selection, were observed in the $\mathrm{N}$ terminal region of CatSper1, we did not find evidence of an association between $\omega$ ratios and levels of sperm competition or sperm velocity parameters. One possible interpretation of these results is that, whereas indel substitutions seem to be a primary target of post-copulatory sexual selection, amino acid divergence may be influenced by multiple selective forces promoting changes not related to reproductive success. On the other hand, it is possible that the different species follow different mutation routes to increase fertilization ability, leading to lineage heterogeneity of adaptive evolution, either within the Catsper1 gene or across multiple genes controlling the same traits. Therefore, an effect of sexual selection on the molecular evolution of CatSper1 cannot be completely discarded despite the fact that our analyses did not reveal such an association.

The role of the $\mathrm{N}$-intracellular region of CatSper1 has not yet been clarified. Catsper1 is a constitutively active unit of the CatSper channel, which is strongly potentiated by intracellular alkalinization [51]. An involvement of the CatSper1 $\mathrm{N}$-terminal region in $\mathrm{pH}$ regulation of the CatSper channel has been suggested because of its high content in histidine residues [51]. Our results showed that variation in the proportion of histidines in the N-terminal region of rodent CatSper1 is not associated to sperm competition levels, sperm velocity or sequence length. Instead, we observed that different murine clades keep a constant amount of histidines, which suggests that successive histidine gains (or losses) have occurred through evolutionary time. Nonetheless, the acquisition (or loss) of histidines in CatSper1 could be adaptive as almost half of the amino acids under positive selection include these residues. On the other hand, previous reports have revealed that the sensitivity of $\mathrm{K}^{+}$channels to intracellular $\mathrm{pH}$ is regulated by histidine residues located in particular intracellular domains [52,53]. It is therefore possible that $\mathrm{pH}$ sensitivity of CatSper1 is related to the location of histidine residues in the $\mathrm{N}$-terminal domain rather than to their abundance.

Another suggested functional model for the intracellular N-terminal region of CatSper1 is one based on the balland-chain model of $\mathrm{K}^{+}$channels [25]. This is plausible because the CatSper channel seems to resemble $\mathrm{K}^{+}$ channels rather than $\mathrm{Ca}^{2+}$ channels. According to the balland-chain model, the N-terminal domain of CatSper would act to physically block the ion channel pore region causing the inactivation of the channel. Variations in length of this intracellular domain could be relevant for the activation/inactivation rate of the channel due to spatial restrictions and, hence, may affect sperm motility [54]. In any case, there could be multiple mechanisms regulating $\mathrm{Ca}^{2+}$ influx through the CatSper channel and further molecular and biochemical approaches clarifying the role of the $\mathrm{N}$-intracellular domain will be necessary to determine the functional consequences of indel and amino acid substitutions in this region as well as which changes may be advantageous in terms of sperm competition.

Our study provides the first evidence of how sperm competition is able to influence traits important for fertilization success by promoting structural changes in a sperm-specific protein. Sperm-specific proteins have evolved through large changes in protein length, with a larger number of indel events, in comparison with genes from other tissues in mammals [55]. Whenever indel substitutions do not imply drastic changes affecting protein function, structural divergence may be a source of variation able to promote advantageous changes more efficiently than nucleotide replacements. Therefore, it is possible that indel substitutions constitute primary targets of positive selection in some reproductive genes.

In any case, caution should be exercised when searching for links between genotypic and phenotypic traits. As mentioned above, in the context of sexual selection, species may exhibit several different mutational routes associated to increase in reproductive fitness. Moreover, many traits evolving under sexual selection are likely to be regulated by multiple genes. Analyses of groups of genes potentially associated with particular phenotypic traits could provide insights of the evolutionary processes of traits important for fertilization.

Our findings also contributed to identify a starting point for future work investigating the influence of evolutionary forces on structural divergence of protein coding sequences. It has been recently discovered that structural variants between close orthologous sequences are rare in the mouse and that a very low proportion of these lead to phenotypic changes [21]. Structural divergence is much more prevalent in duplicated genes, leading to the generation of functionally distinct paralogs [56]. A good example to address these issues may be CatSper itself and, thus, it could be worthwile expanding the present studies to other members of the CatSper gene family.

\section{Conclusions}

Our study has revealed that length variation in the $\mathrm{N}$ terminal region of CatSper1, resulting from an excess of 
indel substitutions favored by positive selection, is associated to relative testes mass, suggesting that sperm competition is a primary force influencing the structural evolution of this intracellular domain of the CatSper channel. In addition, our results have shown that variation in the length of CatSper1 N-terminus is associated with changes in sperm swimming velocity, an essential sperm function for successful fertilization. Altogether, the amino terminal region of CatSper1 seems to be an important target of sexual selection whose structural changes may result in faster sperm which are more likely to win the race to fertilization. To the best of our knowledge, this is the first observation of protein structural divergence linked to evolution of traits important for sperm competition.

\section{Availability of supporting data}

CatSper1 sequences were deposited in GenBank (https:// www.ncbi.nlm.nih.gov/genbank/) with accession numbers KJ652954-KJ652968. Catsper1 nucleotide matrices and resulting phylogenetic trees are available in the TreeBASE repository (http://www.treebase.org) with accession URL: http://purl.org/phylo/treebase/phylows/study/TB2:S15721 [57]. Supporting data are also included as additional files.

\section{Additional files}

Additional file 1: Table S1. Data used for analyses.

Additional file 2: Figure S1. Nucleotide alignment of first exon of Catsper1. Regions containing indel substitutions are indicated. Cricetulus griseus was used as outgroup.

Additional file 3: Figure S2. Catsper1 phylogenetic trees constructed by Neighbor-Joining and Maximum-likelihood methods.

Additional file 4: Figure S3. Regression diagnostics between CatSper1 $\mathrm{N}$-terminus length and relative testes mass.

Additional file 5: Figure S4. Distribution of histidine abundance in CatSper 1 among rodent species.

Competing interests

The authors declare that they have no competing interests.

\section{Authors' contributions}

AV designed and performed the molecular experiments and analyzed data. MT performed the physiological experiments and obtained the phenotypic data. ERSR coordinated the study. AV, MT and ERSR wrote the paper. All authors read and approved the final manuscript.

\section{Acknowledgments \\ This study was funded by the Spanish Ministry of Economy and Competitiveness (grant CGL2011-26341 to ERSR). AV holds a postgraduate scholarship (BES-2009- 029239) supported by the program Formación del Personal Investigador (FPI) whereas MT holds a "Juan de la Cierva" postdoctoral fellowship, both from the Spanish Ministry of Economy and Competitiveness. We thank Lena Lüke for critical revision of the manuscript.}

Received: 15 January 2014 Accepted: 28 April 2014

Published: 16 May 2014

\section{References}

1. Swanson WJ, Vacquier VD: The rapid evolution of reproductive proteins. Genetics 2002, 3:137-144

2. Palumbi SR: All males are not created equal: fertility differences depend on gamete recognition polymorphisms in sea urchins. Proc Natl Acad SCi U S A 1999, 96:12632-12637.

3. Chapman T, Bangham J, Rowe L: Sexual conflict. Evolution 2003, 18:4-6.

4. Birkhead TR, Møller AP: Sperm Competition and Sexual Selection. San Diego, CA: Academic Press; 1998.

5. Parker GA: Sperm competition and its evolutionary consequences in insects. Biol Rev 1970, 45:525-567.

6. Pizzari T, Parker GA: Sperm competition and sperm phenotype. In Sperm biology: an Evolutionary Perspective. Edited by Birkhead TR, Hosken DJ, Pitnick S. Oxford: Academic press; 2009:207-246.

7. Hosken DJ: Experimental evidence for testis size evolution via sperm competition. Science 2001, 4:10-13.

8. Pitnick S, Miller GT, Reagan J, Holland B: Males evolutionary responses to experimental removal of sexual selection. Proc $R$ SOC B 2001, 268:1071-1080.

9. Birkhead TR, Hosken DJ, Pitnick S: Sperm Biology: An Evolutionary Perspective. Oxford: Academic press; 2009

10. Birkhead TR, Mart JG, Burke T, Froman DP: Sperm mobility determines the outcome of sperm competition in the domestic fowl. Proc R Soc B 1999, 266:1759-1764.

11. Gage MJG, Macfarlane CP, Yeates S, Ward RG, Searle JB, Parker GA Spermatozoal traits and sperm competition in Atlantic salmon. Curr Biol 2004, 14:44-47.

12. Froman DP: Sperm mobility: a primary determinant of fertility in the domestic fowl (Gallus domesticus). Biol Reprod 1999, 61:400-405.

13. Malo AF, Garde JJ, Soler AJ, Garcla J, Roldan ERS: Male fertility in natural populations of red deer Is determined by sperm velocity and the proportion of normal spermatozoa. Biol Reprod 2005, 829:822-829.

14. Gomendio M, Roldan ERS: Implications of diversity in sperm size and function for sperm competition and fertility. Int J Dev Biol 2008, 52:439-447

15. Lüpold S, Calhim S, Immler S, Birkhead TR: Sperm morphology and sperm velocity in passerine birds. Proc R Soc B 2009, 276:1175-1181.

16. Fitzpatrick JL, Montgomerie R, Desjardins JK, Stiver KA, Kolm N, Balshine S: Female promiscuity promotes the evolution of faster sperm in cichlid fishes. Proc Natl Acad Sci U S A 2009, 106:1128-1132.

17. Tourmente M, Gomendio M, Roldan ERS: Sperm competition and the evolution of sperm design in mammals. BMC Evol Biol 2011, 11:12.

18. Dorus S, Evans PD, Wyckoff GJ, Choi SS, Lahn BT: Rate of molecular evolution of the seminal protein gene SEMG2 correlates with levels of female promiscuity. Nat Genet 2004, 36:1326-1329.

19. Finn S, Civetta A: Sexual selection and the molecular evolution of ADAM proteins. J Mol Evol 2010, 71:231-240.

20. Lüke L, Vicens A, Serra F, Luque-Larena JJ, Dopazo H, Roldan ERS, Gomendio M: Sexual selection halts the relaxation of protamine 2 among rodents. PLOS One 2011, 6:e29247

21. Yalcin B, Wong K, Agam A, Goodson M, Keane TM, Gan X, Nelláker C, Goodstadt L, Nleod J, Bhomra A, Hernandez-Pliego P, Whitley H, Cleak J, Dutton R, Janowitz D, Mott R, Adams DJ, Flint J: Sequence-based characterization of structural variation in the mouse genome. Nature 2011, 477:326-329.

22. Zichner T, Garfield DA, Rausch T, Stütz AM, Cannavó E, Braun M, Furlong EEM, Korbel JO: Impact of genomic structural variation in Drosophila melanogaster based on population-scale sequencing. Genome Res 2013, 23:568-579.

23. Henrichsen CN, Vinckenbosch N, Zöllner S, Chaignat E, Pradervand S, Schütz F, Ruedi M, Kaessmann H, Reymon A: Segmental copy number variation shapes tissue transcriptomes. Nat Genet 2009, 41:424-429.

24. Schully SD, Hellberg ME: Positive selection on nucleotide substitutions and indels in accessory gland proteins of the Drosophila pseudoobscura subgroup. J Mol Evol 2006, 62:793-802.

25. Podlaha $\mathrm{O}$, Zhang J: Positive selection on protein-length in the evolution of a primate sperm ion channel. Proc Natl Acad Sci U S A 2003, 100:12241-12246

26. Podlaha O, Webb DM, Tucker PK, Zhang J: Positive selection for indel substitutions in the rodent sperm protein Catsper1. Mol Biol Evol 2005 22:1845-1852. 
27. Ramm SA, McDonald L, Hurst JL, Beynon RJ, Stockley P: Comparative proteomics reveals evidence for evolutionary diversification of rodent seminal fluid and its functional significance in sperm competition. $\mathrm{Mol}$ Biol Evol 2009, 26:189-198.

28. Navarro B, Kirichok Y, Chung J, Clapham DE: Ion channels that control fertility in mammalian spermatozoa. Int J Dev Biol 2008, 52:607-613.

29. Qi H, Moran MM, Navarro B, Chong JA, Krapivinsky G, Krapivinsky L, Kirichok Y, Ramsey S, Quill TA, Clapham DE: All four CatSper ion channel proteins are required for male fertility and sperm cell hyperactivated motility. Proc Natl Acad Sci U S A 2007, 104:1219-1223.

30. Marquez B, Ignotz G, Suarez SS: Contributions of extracellular and intracellular $\mathrm{Ca}^{2+}$ to regulation of sperm motility: Release of intracellular stores can hyperactivate CatSper1 and CatSper2 null sperm. Dev Biol 2007, 303:214-221.

31. Alasmari W, Costello S, Correia J, Oxenham SK, Morris J, Fernandes L, Ramalho-Santos J, Kirkman-Brown J, Michelangeli F, Publicover S, Barrat CLR: $\mathrm{Ca}^{2+}$ signals generated by CatSper and $\mathrm{Ca}^{2+}$ stores regulate different behaviors in human sperm. J Biol Chem 2013, 288:6248-6258.

32. Loux SC, Crawford KR, Ing NH, González-Fernández L, Macías-García B Love CC, Varner DD, Velez IC, Choi YH, Hinrichs K: CatSper and the relationship of hyperactivated motility to intracellular calcium and pH kinetics in equine sperm. Biol Reprod 2013, 89(123):1-15.

33. Posada D: jModelTest: phylogenetic model averaging. Mol Biol Evol 2008, 25:1253-1256

34. Steppan SJ, Adkins RM, Anderson J: Phylogeny and divergence-date estimates of rapid radiations in muroid rodents. Sys Biol 2004 53:533-553.

35. Suzuki H, Shimada T, Terashima M, Tsuchiya K, Aplin K: Temporal, spatial, and ecological modes of evolution of Eurasian Mus based on mitochondrial and nuclear gene sequences. Mol Phyl Evol 2004 33:626-646

36. Martín-Coello J, Dopazo H, Arbiza L, Ausió J, Roldan ERS, Gomendio M: Sexual selection drives weak positive selection in protamine genes and high promoter divergence, enhancing sperm competitiveness. Proc $R$ SOC B 2009, 276:2427-2436.

37. Fabre $\mathrm{P}-\mathrm{H}$, Hautier $\mathrm{L}$, Dimitrov $\mathrm{D}$, Douzery EJ: A glimpse on the pattern of rodent diversification: a phylogenetic approach. BMC Evol Biol 2012, 12:88.

38. Yang Z, Nielsen R, Goldman N, Pedersen A: Codon-substitution models for heterogeneous selection pressure at amino acid sites. Genetics 2000, 155:431-449.

39. Swanson WJ, Nielsen R, Yang Q: Pervasive adaptive evolution in mammalian fertilization proteins. Mol Biol Evol 2003, 20:18-20.

40. Yang Z, Wong WSW, Nielsen R: Bayes empirical bayes inference of amino acid sites under positive selection. Mol Biol Evol 2005, 22:1107-1118.

41. Yang Z, Nielsen R: Synonymous and nonsynonymous rate variation in nuclear genes of mammals. J Mol Evol 1998, 46:409-418.

42. Montgomery SH, Capellini I, Venditti C, Barton RA, Mundy NI: Adaptive evolution of four microcephaly genes and the evolution of brain size in anthropoid primates. Mol Biol Evol 2011, 28:625-638.

43. Ramm SA, Oliver PL, Ponting CP, Stockley P, Emes RD: Sexual selection and the adaptive evolution of mammalian ejaculate proteins. Mol Biol Evol 2008, 25:207-219.

44. Kenagy GJ, Trombulak SC: Size and function of mammalian testes in relation to body size. J Mammal 1986, 67:1-22.

45. Freckleton RP, Harvey PH, Pagel M: Phylogenetic analysis and comparative data: a test and review of evidence. Am Nat 2005, 160:712-726.

46. de Jong WW, Rydén $L$ : Causes of more frequent deletions than insertions in mutations and protein evolution. Nature 1981, 290:157-159.

47. Xia J, Reigada D, Mitchell CH, Ren D: CATSPER channel-mediated $\mathrm{Ca}^{2+}$ entry into mouse sperm triggers a tail-to-head propagation. Biol Reprod 2007, 77:551-559.

48. Ho K, Wolff CA, Suarez SS: CatSper-null mutant spermatozoa are unable to ascend beyond the oviductal reservoir. Reprod Fertil Dev 2009, 21:345-350.

49. Ren D, Navarro B, Perez G, Jackson AC, Hsu S, Shi Q, Tilly JL, Clapham DE: A sperm ion channel required for sperm motility and male fertility. Nature 2001, 413:603-609.

50. Gómez Montoto L, Varea Sánchez M, Tourmente M, Martín-Coello J, Luque-Larena JJ, Gomendio M, Roldan ERS: Sperm competition differentially affects swimming velocity and size of spermatozoa from closely related muroid rodents: head first. Reproduction 2011, 142:819-830.
51. Kirichok Y, Navarro B, Clapham DE: Whole-cell patch-clamp measurements of spermatozoa reveal an alkaline-activated $\mathrm{Ca}^{2+}$ channel. Nature 2006, 439:737-740.

52. Coulter KL, Perier F, Redeke CM, Vanderberg CA: Identification and molecular localization of a $\mathrm{pH}$-sensing domain for the inward rectifier potassium channel HIR. Neuron 1995, 15:1157-1168.

53. Chanchevalap S: Involvement of histidine residues in proton sensing of ROMK1 channel. J Biol Chem 2000, 275:7811-7817.

54. Hoshi T, Zagotta WN, Aldrich RW: Biophysical and molecular mechanisms of shaker potassium channel inactivation. Science 1990, 250:533-538.

55. Torgerson DG, Kulathinal RJ, Singh RS: Mammalian sperm proteins are rapidly evolving: evidence of positive selection in functionally diverse genes. Mol Biol Evol 2002, 19:1973-1980.

56. Xu G, Guo C, Shan H, Kong H: Divergence of duplicate genes in exon-intron structure. Proc Natl Acad Sci U S A 2011, 109:1187-1192.

57. Vicens A, Tourmente M, Roldan ERS: Sequence matrix and phylogenetic trees of the first exon of Catsper1 of murid rodents. TreeBASE 2014, http://purl.org/phylo/treebase/phylows/study/TB2:S15721.

doi:10.1186/1471-2148-14-106

Cite this article as: Vicens et al: Structural evolution of CatSper1 in rodents is influenced by sperm competition, with effects on sperm swimming velocity. BMC Evolutionary Biology 2014 14:106.

\section{Submit your next manuscript to BioMed Central and take full advantage of:}

- Convenient online submission

- Thorough peer review

- No space constraints or color figure charges

- Immediate publication on acceptance

- Inclusion in PubMed, CAS, Scopus and Google Scholar

- Research which is freely available for redistribution

Submit your manuscript at www.biomedcentral.com/submit
C BioMed Central 